\title{
Automatic segmentation of Colon Cancer Cells Based on Active Contour Method: A New Approach
}

Jamal Charara, Alaa Hilal, Ali Al Houseini, Walid Hassan and Mohamad Nassreddine.

Department of Physics and Electronics, Faculty of Sciences I, Lebanese University, Beirut, Lebanon

Received: September 28, 2012 / Accepted: December 7, 2012 / Published: February 28, 2013.

\begin{abstract}
Automatic interpretation of the images of colon cell biopsies requires automatic segmentation of these cells in the image obtained. The active contour method for image segmentation is a well known method for automatic detection of the cell contour. However, the application of this method on colon cell images was not effective. In this paper, the authors have proposed a new technique to reduce the analysis time needed to detect cells in a given image. This technique is based on the active contour method but now using a progressive division of the dimensions of the image to achieve convergence. The model proposed succeeded in detecting cells whose boundaries are not necessarily defined by a gradient. The initial curve can be anywhere in the image, and interior contours can be automatically detected. The developed algorithm was successfully applied on textured multispectral images of three types of cells, including benign hyperplasia (BH), intraepithelial neoplasia (IN), and carcinoma (Ca) cells.
\end{abstract}

Key words: Active contours, multispectral image, texture, segmentation.

\section{Introduction}

Medical images obtained microscopically are important tools in medical diagnosis. Since colon cancer, like any other cancer, is characterized by abnormal cellular proliferation, seeking abnormalities inside microscopic images from biopsies, such as elliptical non-regular cancer cells or non-natural contrast, is of paramount interest. However, diagnosis based on observation of medical images would be largely accelerated if the manual search of abnormalities can be replaced by an automatic procedure. The majority of methods used to classify cancerous cells use morphological image processing, particularly the shapes of the cells inside images. The effectiveness of an automatic search method is generally assessed by its capacity to analyze and interpret a large number of images in a short time. The main drawback of these approaches is the long analysis time [1].

The active contour (snake) method is a very effective technique for rapid segmentation of cancer cells in the image. It is based on color intensity and can be applied on sequences of objects in the image. Nevertheless, segmentation of colon cancer cells possessing irregular shapes within a multispectral image was not efficient with the active contour [2-4].

The objective of the present paper was to develop a new approach aiming to reduce the time necessary to detect cells in a given image. This approach was derived from the active contour method but now using a progressive division of the dimensions of the image to achieve convergence. Three types of cells were utilized to assess the efficiency of our segmentation model, including benign hyperplasia (BH), intraepithelial neoplasia (IN) that is a precursor state for cancer, and carcinoma ( $\mathrm{Ca}$ ) that corresponds to abnormal tissue proliferation (cancer).

\section{Materials and Methods}

\subsection{Active Contour Model}

Active contour is a dynamic curve that tends, in an 
iterative process, to move toward and detect the contour of an object observed in a certain image. This curve consists of a set of points connected to each other. An energy function is generally associated with this curve (snake) in such a way:

$$
F_{\text {snake }}=F_{\text {internal }}+F_{\text {external }}
$$

where $F_{\text {internal }}$ is an energy that depends on the physical properties of the contour and $F_{\text {external }}$ is another energy that depends on the properties of the image $[3,5,6]$. The corresponding algorithm tends to search for a combination between different image points in order to minimize the energy function $F_{\text {snake }}$ and thus detect the contour.

The model have developed was able to detect the contour of an image without calculating its gradient and/or detecting its edges [7].

Fig. 1 represents an image of a single object having more intensity different than that of the background.

$c_{1}$ and $c_{2}$ are the average intensities in the regions inside and outside $C_{0}$, respectively. The energy $F$ is defined as:

$F=F_{1}(C)+F_{2}(C)=\sum_{\text {interior }(C)}\left|U_{0}(x, y)-c_{1}\right|^{2}+$
$\sum_{\text {exterior }(C)}\left|U_{0}(x, y)-c_{2}\right|^{2}$

According to $F$, four different cases of contour detection are possible as shown in Fig. 2. These four cases consider all possible outcomes of the green contour with the real one as follows [4]:

$$
\begin{aligned}
& F_{1}(C)>0 \text { and } F_{2}(C) \approx 0 \\
& F_{1}(C) \approx 0 \text { and } F_{2}(C)>0 \\
& F_{1}(C)>0 \text { and } F_{2}(C)>0 \\
& F_{1}(C) \approx 0 \text { and } F_{2}(C) \approx 0
\end{aligned}
$$

It can also add to $F$ a regularization term that leads to the final expression of $F$ :

$F=\mu$. length $(C)+\sum_{\text {interior }(C)}\left|U_{0}(x, y)-c_{1}\right|^{2}+$

$$
\sum_{\text {exterior }(C)}\left|U_{0}(x, y)-c_{2}\right|^{2}
$$

where $\mu \geq 0$ is a fixed parameter. Consequently, the detection of contours simplifies to become the solution of:

$$
\underbrace{\inf _{1}}_{c_{1}, c_{2}, \mathrm{C}}\left(c_{1}, c_{2}, C\right)
$$

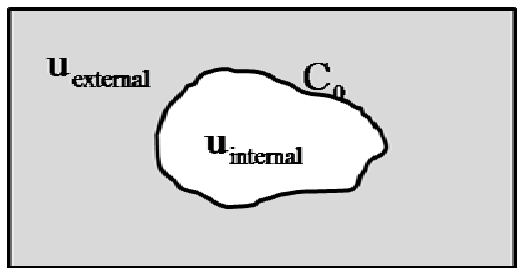

Fig. 1 Image representing a single object with uniform intensity $U_{\text {internal }}$, separated by the contour $C_{0}$ from its back ground with uniform intensity $U_{\text {external }}$.

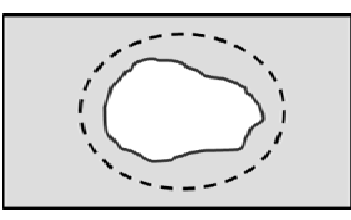

(a)

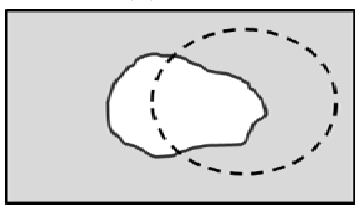

(c)

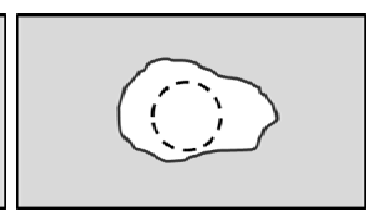

(b)

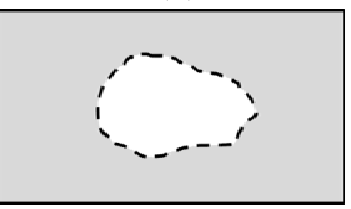

(d)
Fig. 2 Four different cases of contour detection.

\subsection{Adaptation of the Active Contour Method}

The approach is derived from the active contour model. It leads to effective and fast medical image segmentation in particular segmentation of high dimensional images. Indeed, it consists of a set of eight consecutive segmentation steps. In the first step, the dimension of the image is reduced to $12.5 \%$ of its original size. This means that for an initial image of $512 \times 512$ pixels, the dimension of the image of the first step $I_{1}$ is reduced to $64 \times 64$ pixels. Segmentation of the images begins with an initial active square contour of dimension $42 \times 42$ pixels. $C_{1}$ is the contour resulting from the first segmentation step. In the second segmentation step, the dimension of the image is reduced to $25 \%$ of its original size. Thus, the dimension of the image $I_{2}$ of the second step becomes $128 \times 128$ pixels and the contour $C_{1}$, resulting from the first segmentation step, is used as an initial contour to segment the image $I_{2}$. The contour $C_{2}$ obtained after this segmentation is resized and used to segment the image $I_{3}$ of the third step. 
Automatic Segmentation of Colon Cancer Cells

Based on Active Contour Method: A New Approach

Similarly, it continues with the remaining steps to finally segment the initial $512 \times 512$ pixels image. At each step, the initial image is reduced to the corresponding dimension (image $I_{i}$ ) and the contour obtained by the previous segmentation step $\left(C_{i-1}\right)$ is resized to $I_{i}$ 's size and used as initial contour for segmentation. Fig. 3 shows the segmentation procedure scheme.

This progressive segmentation of the image is performed automatically. A condition can be imposed on the number of iterations necessary to reach convergence. In this case, a set of 20 consecutive iterations was allowed for each segmentation step. It measures for each step the vector $D_{0}$ that is the spatial distance between two consecutive contours of the 20 iterations. In addition, we measure, at the end of each step, the vector element $D_{s i}$ that is the difference between the maximum and the minimum of vector $D_{0}$. The first step (first twenty iterations) is characterized to have $D_{s 1}$ as the maximum value of $D_{s i}$. This procedure continues in performing 20 new iteration steps until:

$$
D_{s i}<\alpha . D_{s 1}
$$

where $\alpha$ is a parameter that depends on the sensitivity of the required segmentation and is introduced by the user. However, an analysis of the curve obtained at each step can give an idea about the order of the $\alpha$ parameter such as:

$$
\alpha=\min \left(D_{s i}\right) / D_{s 1}
$$

\section{Results and Discussion}

Fig. 4 shows the result after 6 min of segmentation per image of the three types of cells ( $\mathrm{BH}, \mathrm{IN}$ and $\mathrm{Ca})$ using the classical active contour segmentation method. All of these contours are far from the real contour of each cell.

Fig. 5 represents the images of the three types of cells $(\mathrm{BH}, \mathrm{IN}$, and $\mathrm{Ca}$ ) resized to the dimension $64 \times$ 64 pixels. This figure shows also that successful segmentations were obtained within 2-4 s.

However, the developed algorithm showed that beyond a threshold number of iterations, the active contour of the model fluctuates around the real geometry in a repeated manner (Fig. 6). This

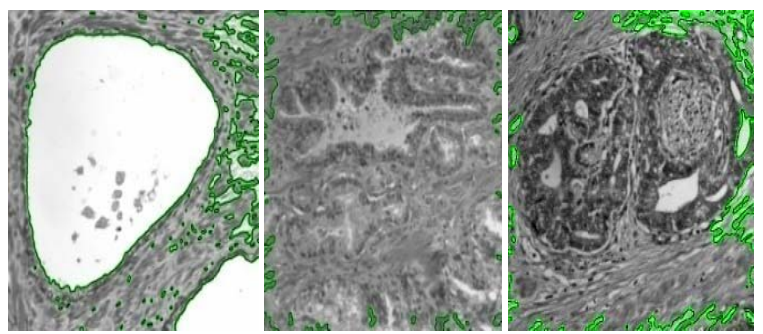

Fig. 4 Segmentation results of three images of dimension $512 \times 512$ pixels using classical active contour model.

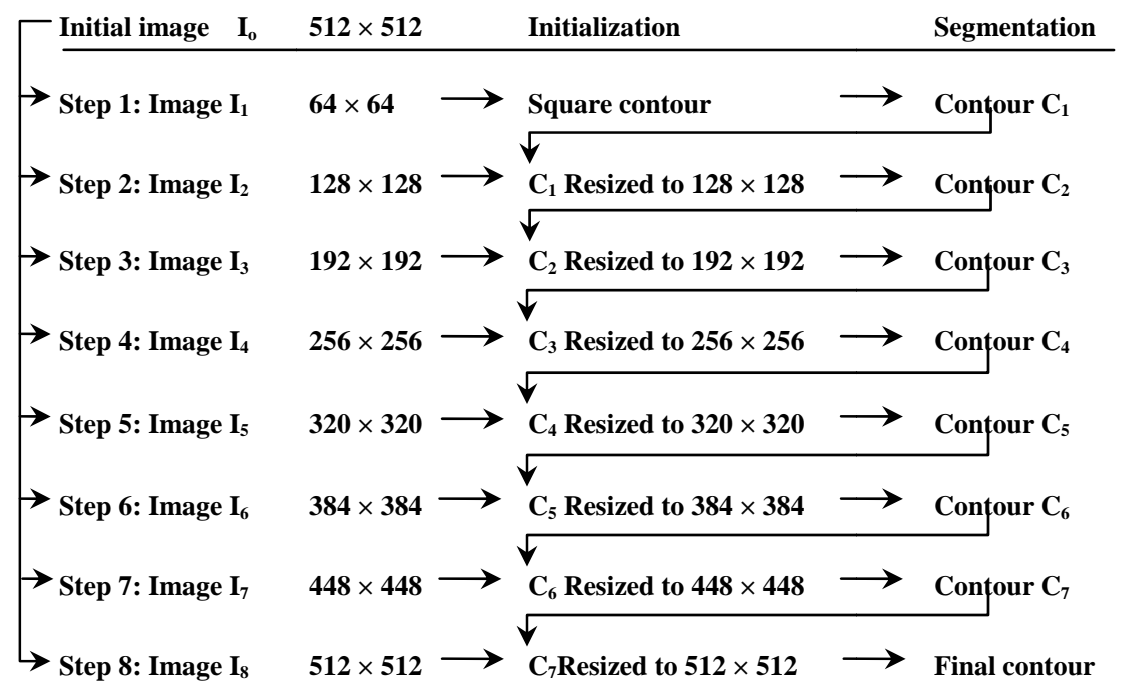

Fig. 3 The eight steps of the segmentation process. 

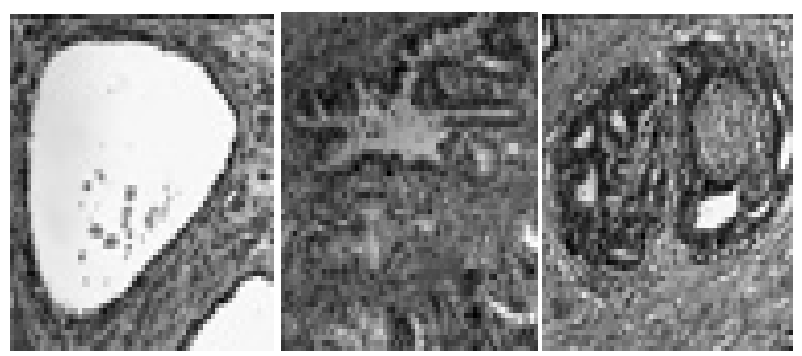

(a)
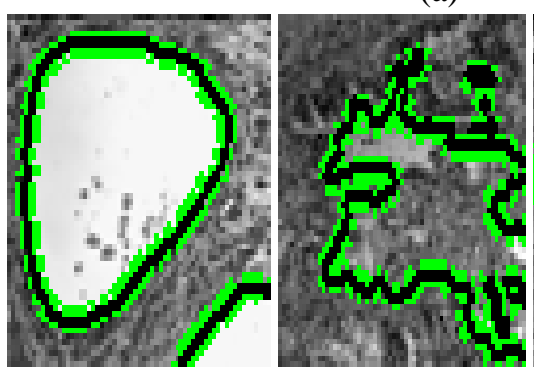

(b)

Fig. 5 (a) The three types of colon cells are reduced to $64 \times$ 64 pixels; (b) Segmentation results of the three images of size $64 \times 64$ pixels.

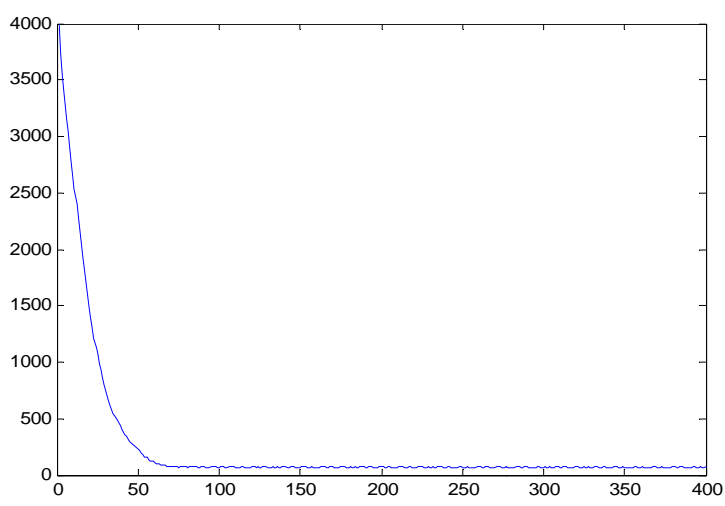

Fig. 6 Constant fluctuation of the contour after 75 iterations.

fluctuation increases the processing time without any improvement of the detected contour.

Table 1 shows the values of $\alpha$ parameter for the eight steps of segmentation of the image illustrated in Fig.5. The value of $\alpha$ varies between $0.2 \%$ and $1.7 \%$. This value, that ensures convergence, must be unique for the eight segmentation steps. Fig. 7 shows the variations of $\alpha$ for segmentation steps 1 and 2. Also it shows that as the resolution of the image increases the number of iterations necessary to achieve convergence decreases.

Fig. 8 shows complete segmentation of full size images (512 $\times 512$ pixels) of Fig 4 with $\alpha=5 \%$. The average processing time was $147.1969 \mathrm{~s}(\approx 2.4533$ $\min )$. This figure shows the efficiency of the model in detecting active contours of irregular objects such as cancerous cells of types IN and Ca.

\section{Conclusion}

The effectiveness of the technique was based on the progressive segmentation of the image. The model led to two major consequences. First time consumption of the segmentation process was shortened to less than 3

Table $1 \alpha$ values for the eight segmentation steps.

\begin{tabular}{lccll}
\hline Step & Image Size & Min & Max & $\mathbf{( \% )}$ \\
\hline 1 & $64 \times 64$ & 7.031 & 410 & 1.7 \\
2 & $128 \times 128$ & 15.4 & 2,08 & 0.6 \\
3 & $192 \times 192$ & 14.83 & 2,803 & 0.5 \\
4 & $256 \times 256$ & 7.596 & 2,944 & 0.2 \\
5 & $320 \times 320$ & 49.56 & 2,944 & 1.6 \\
6 & $384 \times 384$ & 24.19 & 3,149 & 0.7 \\
7 & $448 \times 448$ & 38.55 & 3,201 & 1.2 \\
8 & $512 \times 512$ & 43.32 & 3,289 & 1.4 \\
\hline
\end{tabular}
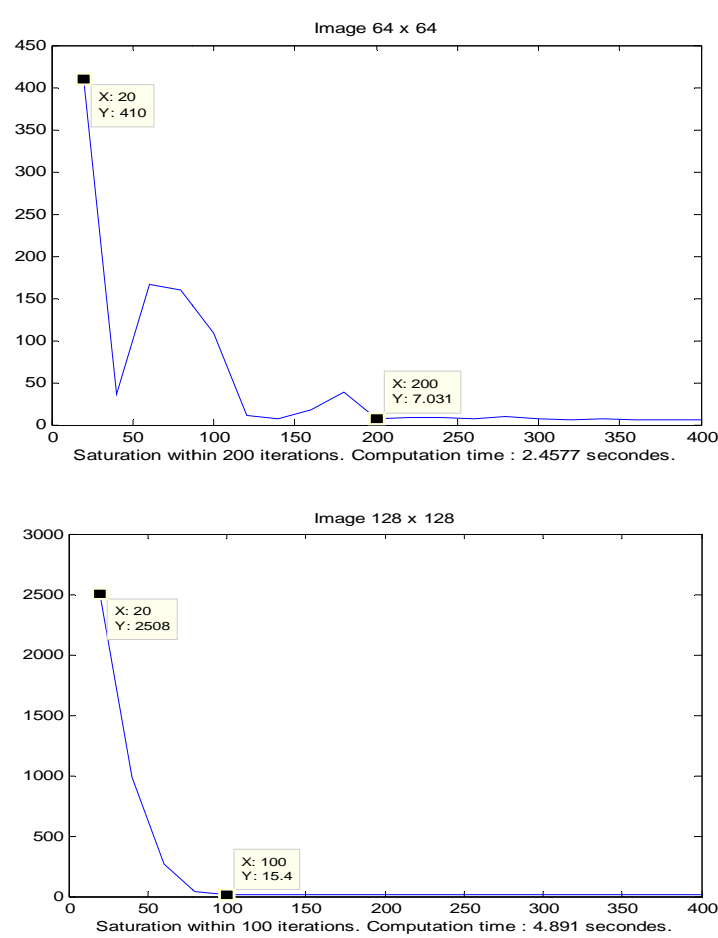

Fig. 7 Analysis of $\alpha$ for segmentation steps 1 and 2. 
Automatic Segmentation of Colon Cancer Cells Based on Active Contour Method: A New Approach

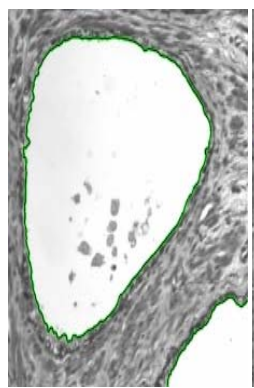

(a)

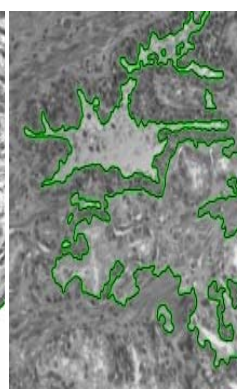

(b)

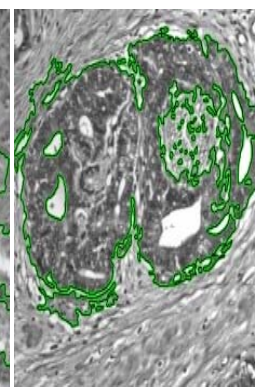

(c)
Fig. 8 Time consumption and segmentation results for the three types of colon cells: (a) BH: $164.74 \mathrm{~s}$; (b) IN: $136.98 \mathrm{~s}$ and (c) Ca: $139.87 \mathrm{~s}$.

minutes per image. Second, the segmentation of colon cancer cells has become fully automated. Therefore, the proposed model allows accurate and efficient segmentation of images containing distinct objects in a very short time. The approach is very simple and exhibits attractive results. This method is useful in the automatic segmentation between different histopathological images and thus allowing a faster segmentation of microscopic bio-images.

\section{Acknowledgments}

This work was supported by grants from the Doctoral School of Sciences \& Technology at the Lebanese University.

\section{References}

[1] C.A.B. Castano, J.S. Fraga, S. Fernandez, A. Gruber, L.
Costa, Biological shape characterization for automatic image recognition and diagnosis of protozoan parasites of the genus Eimeria, Pattern Recognition 40 (2007) 1899-1910.

[2] T.E. Schneider, Automated classification of analysis and reference cells for cancer diagnostics in microscopic images of epithelial cells from the oral mucosa, Acta Polytechnica 47 (2007) 86-90.

[3] G.D. Giannoglou, Y.S. Chatzizisis, V. Koutkias, I. Kompatsiaris, M. Papadogiorgaki, V. Mezaris, et al., A novel active contour model for fully automated segmentation of intravascular ultrasound images: In vivo validation in human coronary arteries, Computers in Biology and Medicine 37 (2007) 1292-1302.

[4] R. Verdu, J. Morales, R. Gonzalez, L. Weruaga, Convergence analysis of active contours, Image and Vision Computing 26 (2008) 1118-1128.

[5] R.M. Haralick, K. Shanmugam, I. Dinstein, Textural features for image classification, IEEE Transactionson Systems, Man and Cybernetics 3 (1973) 610-621.

[6] T. Chan, L. Vese, Active contours without edges, IEEE Transactions on Image Processing 10 (2001) 266-277.

[7] D. Mayumi, U. Sabino, L.F. Costa, E.G. Rizatti, M.A. Zago, A texture approach to leukocyte recognition, Real-Time Imaging 10 (2008) 205-216.

[8] M.A. Roula, A. Bouridane, P. Miller, Aquadratic classifier based on multi-spectraltexture features for prostate cancer diagnosis, in: Proceedings of the 7th International Symposium on Signal Processing and its Applications, 2003, pp. 37-40.

[9] M.A. Garcia, D. Puig, Supervised texture classification by integration of multiple texture methods and evaluation windows, Image and Vision Computing 25 (2007) 1091-1106. 